\title{
TOUCH BASED AGE ANALYZATION ON MOBILE PHONES USING MACHINE LEARNING ALGORITHMS
}

\author{
B. Dinesh Reddy, Thotakura Madhuri, N. Thirupathi Rao \\ and Debnath Bhattacharyya \\ Department of Computer Science and Engineering \\ Vignan's Institute of Information Technology (A) \\ Visakhapatnam 530049, AP, India \\ dinesh4net@gmail.com, t.madhu596@gmail.com, \\ nakkathiru@gmail.com,debnathb@gmail.com
}

\begin{abstract}
Smart devices are wherever these days, as Android is an open and free Linux based working framework, generally utilized for cell phones like advanced mobile phones and tablets, where the Android assumes a predominant job in this portable period. In this article, it depicts singular age dependent upon the age of the individual we get the pertinent program data. The preparing of this information or data obtained from the application is secured in a server. From the server we separate the data and after that we train the model by using Machine Learning in order to anticipate the data i.e., we analyze the person's age.
\end{abstract}

\section{Keywords - Android, Server, Model, Machine Learning, Dendrogram, Event}

\section{INTRODUCTION}

"Android" alludes to the robot. We presently realize that it is the Google's open source versatile working framework. Android depends on Linux stage, and comprises of a working framework, middleware, UI, and application programming, which is known as the primary really open portable terminal programming [1]. In order to develop an application, we need to choose the essential and its sections in that particular development. Further, we make sense of which class or in its techniques; we are isolating to develop the application. The data that is procured from the application is removed and the dataset is given to the model in order to set it up. In the wake of setting up the model we by then apply Prediction count to anticipate the data as demonstrated by the dataset that we have given to the model.

\section{LITERATURE REVIEW}

In the current section, some related works that had done so far with the currently considered models are presented as follows:

Ibrahim Mousa Al-Zuabi et. al., [12] had discussed in detail about the records of the details and customer relationships and their management with the combination of billing amounts as a data source for the identification of behaviour of the customers. They had used various machine learning algorithms such that to provide the details about the marketing business strategies, business applications and customer related likes and

Received: May 10, 2019

Reviewed: August 21, 2019

Accepted: August 27, 2019 
dislikes. They had provided the results in such a way that the people using by the model was given as $65 \%$ based on the age prediction of the users.

Nisreen Ameen and Robert Willis [13] had discussed about the usage or the much more using of mobile phones with various age groups among the people in the UAE. They had proposed the method such that extensions to the existing model of the methods like the UTAUT2 model. Several set of questions were posed to the users of the mobile phones and their gadgets in Dubai and around and the results were presented. The results show that there is a different opinion on the users of the mobile phones in the age groups from 18-25 to the other group of 25-35 groups.

Leah Findlater et. al., [14] had studied on touch screen effects for various age groups especially for the older adults. They had compared the performance of the effects between the age groups older adults and younger adults on various touch screen materials. The points considered are crossing, dragging, clicking, pointing etc. The results show that the utilization of the touch screen for the young adults is better than the older adults.

Asim Sinan Yuksel et. al., [15] had analyzed on the topics of identification of people who were using smartphones at various ages. They had considered various algorithms to analyze and given a comparative analysis like the neural networks and support vector machines etc. The results are displayed in the results section with a comparison of both the considered modeled algorithms.

Nicholas D. Lane et. al., [16] had given a detailed review on the various types of Smartphone available in the market and various types of methods to be followed to identify the people who were using these smartphones at various age groups.

From all these review works and previous works, touch based age identification was not completely done by the existing works. Hence, in the current work an attempt has been made to identify the age of a person or age group of a particular persons by touching the screen of a smartphones. The idea was new to some extent for the identification of the persons age and by suing the machine learning algorithms an attempt has been made and the results were encouraging to identify the persons age based on touch of the smartphones screen by a person.

\section{METHODOLOGY}

In Order to achieve the considered method and models, several steps to be followed to achieve the considered output. Hence, the detailed analysis on the steps considered and the performance of such models are discussed as follows,

\section{A. THE STUDY OF MOTIONEVENT CLASS IN DEVELOPING AN APPLICATION:}

Motion event occasions depict reaction code developments and a lot of hub esteems. The activity code indicates the change all through the express the happen like a downward or upward pointer. The estimations of a hub clarify the position and different properties of the development. The axis values clarify the position and other development properties. For instance, when the client first contacts the screen, the framework incorporates the pertinent View with a touch occasion with the activity code ACTION_DOWN and a lot of hub esteems including the $\mathrm{X}$ and $\mathrm{Y}$ directions of the touch and data about the contact territory's weight, size and introduction. A portion of the gadgets can at the same time report different hints of development. For each finger, multicontact screens can emanate one movement follow. Pointers are alluded to as individual fingers or different items that produce hints of development. Movement occasions contain data pretty much all at the present working pointers despite the fact that a large portion of them have not moved since the last occasion was conveyed [2]. 
Each pointer has an outstanding id selected when it goes down fundamentally. Each pointer list in the event ranges from 0 to 1 not actually the estimation of getPointerCount().So as to play out a movement between the gets, we use OnClickListener to get click on the gets along the edge of the analyst organize. We will be facilitated to the inspector organize dependent upon the choice of our catch. Furthermore, in specific events we can use OnTouchListener anyway we can't OnTouchListener at top notch in light of the way that it can't understand gets now and again [2].

\section{B. CONNECTING APPLICATION WITH SERVER}

Here's the XAMPP Server we are using. XAMPP server full structure is $\mathrm{X}$ speaks to Cross-organize, (A)Apache server, (M)MariaDB, (P)PHP and (P)Perl. By and large the Cross-arrange infer that it can continue running with any working structure on any PC. Additionally, it is alocal have or an area server. In solitude work territory or workstation telephone neighborhood has server works. This XAMPP server programming empowers you to test MYSQL, PHP, Apache and Perl stretches out on our neighborhood PC in a legitimate area [3].

Next MariaDB is the most standard database server and MYSQL pack makes it. PHP now and again gives web improvement space. PHP is a language of scripting on the server side. In like way, the last Perl is a programming language that is used to develop a web application. XAMPP server joins mechanical get-togethers, for instance, Apache, MYSQL, PHP, and Perl gadgets. MYSQL, PHP, and Apache are the mechanical gatherings we used in our paper [3]. We partner the Mobile hotspot with our PC to make localhost association or close to server union. When we are using the Application wherever we address the Mobile Phone the data will be customarily at the zone server. In the end, we run the Apache and MYSQL in the XAMPP server control board and make MYSQL as executive it is showed up in Figure 1 [4].

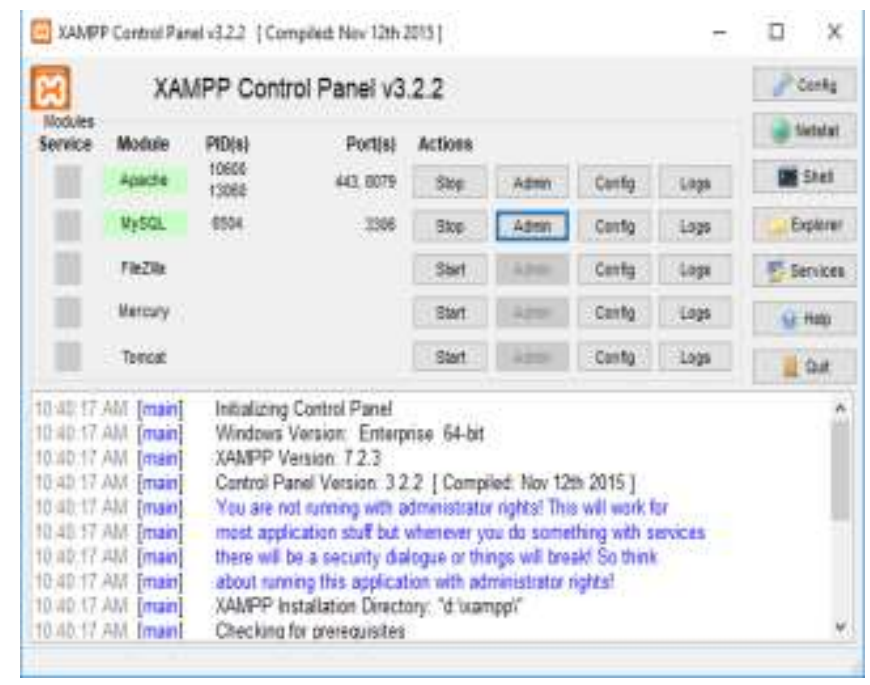

Fig. 1 XAMPP Server Connection

Next, we proceed onward into the following page i.e., the neighborhood have page we embed the quantity of sections required for recovering the information from MYSQL and at the opposite side in the server, we compose the PHP code and keep it in htdcos for executing the quantity of segments required through which we can undoubtedly go on with MYSQL and actualize it i.e., naming of segments for database to get precise information relying on of size, amount characterized, and further we decide the segment information types. After this is done we can send out the information into .CSV 
document. Whatever, information that we get from the application i.e., taken from the server is currently given as an information document to the Machine Learning so as to foresee the information $[5,6]$.

\section{MACHINE LEARNING}

AI is a man-made brainpower field that utilizes factual methods to give PC frameworks the capacity to "learn" (e.g., progressively improving execution on an exact undertaking) from information, without open programming [5]. AI is firmly identified with (and frequently covers with) PC measurements, which additionally centers on foreseeing the utilization of PCs from side to side. It has solid connections to numerical enhancement, giving the field strategies, hypothesis and application areas. AI is now and again connected to information mining, where the last subfield is progressively centered around exploratory information investigation and is known as unsupervised learning. These expository models empower specialists, information researchers, architects, and experts to "produce dependable, repeatable choices and results" and find "shrouded bits of knowledge" by gaining from authentic connections and information patterns. Here, we center on forecast in our paper.

\section{K- MEANS CLUSTERING ALGORITHM:}

$K$-means clustering is a type of unsupervised learning, which is used when you have unlabeled data (i.e., data without defined categories or groups). The goal of this algorithm is to find groups in the data, with the number of groups represented by the variable $K$. Data points are clustered based on feature similarity. The results of the $K$-means clustering algorithm are:

1. The centroids of the $K$ clusters, which can be used to label new data

2. Labels for the training data (each data point is assigned to a single cluster)

Clustering is one of the most common exploratory data analysis technique used to get an intuition about the structure of the data. It can be defined as the task of identifying subgroups in the data such that data points in the same subgroup (cluster) are very similar while data points in different clusters are very different. It combines the ant colony optimization algorithm with condensed hierarchical clustering algorithm, proposes a hybrid clustering method HCAA, using the intelligent ant colony optimization algorithm for the global optimal. But it only uses Euclidean distance as the similarity measurement, so the calculation accuracy is low [7].

\section{K- MEANS ALGORITHM:}

The K- Means algorithm works as follows:

1. Specify number of clusters $K$.

2. Initialize centroids by first shuffling the dataset and then randomly selecting $K$ data points for the centroids without replacement.

3. Keep iterating until there is no change to the centroids. i.e., assignment of data points to clusters isn't changing.

- Compute the sum of the squared distance between data points and all centroids.

- Assign each data point to the closest cluster (centroid).

- Compute the centroids for the clusters by taking the average of the all data points that belong to each cluster.

The objective function is defined for $\mathrm{k}$ - means algorithm as follows:

$$
J=\sum_{i=1}^{m} \sum_{k=1}^{K} w_{i j}\left\|x^{i}-\mu_{k}\right\|^{2}
$$


where $w_{i j}=1$ for data point xi if it belongs to cluster $k$; otherwise, $w_{i k}=0$. Also, $\mu_{k}$ is the centroid of $x^{i}$, s cluster.

The K-Means algorithm typically uses Euclidean or squared Euclidean distance to measure the distortion between a data object and its cluster centroid [8]. The Euclidean and squared Euclidean distances are usually computed from raw data and not from standardized data. This paper proposes a modified K-Means algorithm, by applying Minmax normalization procedure [10] and Max-min distance measure [9] to reach better performance. The Euclidean distances are usually computed from raw data and not from standardized data [11]. It is determined as:

$$
d\left(t_{i}, t_{j}\right)=\sqrt{\sum_{k=1}^{d}\left(t_{i k}-t_{j k}\right)^{2}}
$$

\section{AGGLOMERATIVE HIERARCHICAL CLUSTERING ALGORITHM:}

The Algorithm is as follows:

1. Compute the proximity matrix

2. Let each data point be a cluster

3. Repeat: Merge the two closest clusters and update the proximity matrix

4. Until only a single cluster remains

Key operation is the computation of the proximity of two clusters. This can be visualized by using the Dendrogram, which is a tree-like diagram that records the sequences of merges or splits.

Figure 3 represents the Proposed Model as we have built up an application in that first we have to enter our age and afterward, the diversion begins. Whatever operations are performed on the application, the data is stored in the server i.e., XAMPP server by using this server we save the data which is the number of touches like single finger touch or multi-finger touch and the time elapsed for the touch on the application. We take all qualities and it will be put away in the XAMPP server from the XAMPP server we will trade esteems by utilizing the .csv document. 


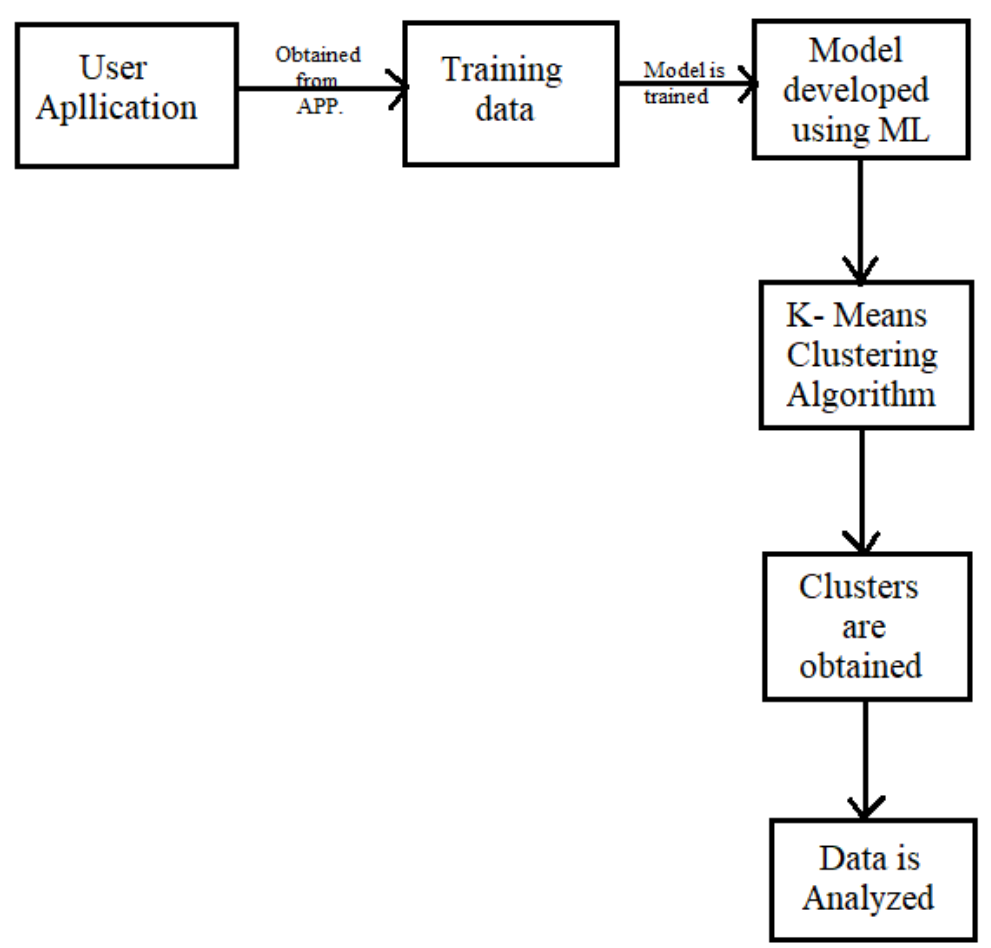

Fig. 3 Architecture of Proposed Model

As the data is obtained from the server we then train the model by using Machine Learning. Before we train the model we find average time elapsed according to the person's age then the data is trained without any bulk of data for similar age group person. In Machine Learning we are using K- Means Clustering Algorithm as it is intended to partition $\mathrm{n}$ objects into k clusters in which each object belongs to the cluster with the nearest mean. This method produces exactly $\mathrm{k}$ different clusters of the greatest possible distinction. By using this algorithm we analyze the person age if the person is having more number of multi-touches then the personage is below 15, if the person time elapse is more on his touch then his age will be above 55 else the person age will be between 15 to 55 .

\section{RESULTS AND DISCUSSION}

\section{A. DATASET}

The dataset that we are taking is gotten from the Application that we had created utilizing the Android Studio utilizing the MotionEvent class in the improvement of Application. Figure 4 speaks to the dataset. This is the data that we are taken for training the model using the Machine Learning Algorithm in order to analyze the data i.e., to estimate the age of the person.

\begin{tabular}{|c|c|c|c|c|c|}
\hline S1.No & Multi & Age & Xposition & Yposition & Time count \\
\hline 1 & 4 & 4 & 297.6799 & 70.46167 & 1 \\
\hline 2 & 4 & 4 & 384.5995 & 427.432 & 2 \\
\hline 3 & 4 & 4 & 533.7391 & 68.5752 & 1 \\
\hline 4 & 4 & 4 & 233.7391 & 168.5752 & 1 \\
\hline 5 & 1 & 5 & 685.7835 & 20.60022 & 2 \\
\hline 6 & 2 & 5 & 454.209 & 3174.388 & 1 \\
\hline
\end{tabular}




\begin{tabular}{|l|l|l|l|l|l|}
\hline 7 & 2 & 5 & 295.6818 & 69.46228 & 2 \\
\hline 8 & 2 & 5 & 881.1397 & 100.446 & 2 \\
\hline 9 & 1 & 5 & 904.1184 & 56.46899 & 2 \\
\hline 10 & 0 & 34 & 295.6818 & 69.46228 & 1 \\
\hline 11 & 0 & 34 & 881.1397 & 100.446 & 1 \\
\hline 12 & 0 & 34 & 384.5995 & 127.432 & 1 \\
\hline 13 & 0 & 34 & 233.7391 & 168.5752 & 1 \\
\hline 14 & 1 & 5 & 233.7391 & 468.5752 & 1 \\
\hline 15 & 2 & 5 & 185.7835 & 20.60022 & 1 \\
\hline 16 & 2 & 5 & 954.209 & 1174.388 & 1 \\
\hline 17 & 2 & 5 & 395.6818 & 69.46228 & 1 \\
\hline 18 & 3 & 6 & 881.1397 & 1200.446 & 1 \\
\hline 19 & 2 & 6 & 495.6818 & 69.46228 & 2 \\
\hline 20 & 0 & 65 & 881.1397 & 100.446 & 3 \\
\hline 21 & 0 & 65 & 395.6818 & 69.46228 & 1 \\
\hline 22 & 0 & 65 & 684.5995 & 177.432 & 3 \\
\hline 23 & 0 & 65 & 233.7391 & 681.5752 & 4 \\
\hline 24 & 0 & 65 & 233.7391 & 618.5752 & \\
\hline
\end{tabular}

Fig. 4 Dataset

This is whole set of dataset that we have obtained from the user application i.e., Android application got the data through XAMPP server. From the XAMPP server we retrieve the data. Here, we take only the required fields like multi, time count and age from the whole set of the dataset, remaining all are used for our considerations.

\begin{tabular}{|c|c|}
\hline Age & Time count \\
\hline 1 & 0 \\
\hline 2 & 0 \\
\hline 3 & 1.58 \\
\hline 4 & 1.63 \\
\hline 5 & 1.97 \\
\hline 6 & 1.62 \\
\hline 7 & 1.52 \\
\hline 8 & 1.42 \\
\hline 9 & 1.64 \\
\hline 10 & 1.52 \\
\hline 11 & 1.13 \\
\hline 12 & 1.09 \\
\hline 13 & 0.98 \\
\hline 14 & 1.04 \\
\hline
\end{tabular}

Fig. 5 Average time count of overall dataset

Figure 5 represents the average time count of overall dataset according to their age groups this done by implementing using python code to it. By taking whole dataset and applying the python programming to the dataset we have chosen the field likes age and time count.

\section{B. CLUSTERS}

From the above dataset of Figure 4, we have represented it in graphical format as shown in Figure 6.1 which represent the multi-touch is present in the age group below 15 years and sometimes observed above the age 55years. From the Average time count of overall dataset i.e., Figure 5, we have applied K- Means Clustering Algorithm and 
obtained clusters as shown in Figure 6.2 represents the average time count. Figure 6.3 represents the multi and time count clustering.

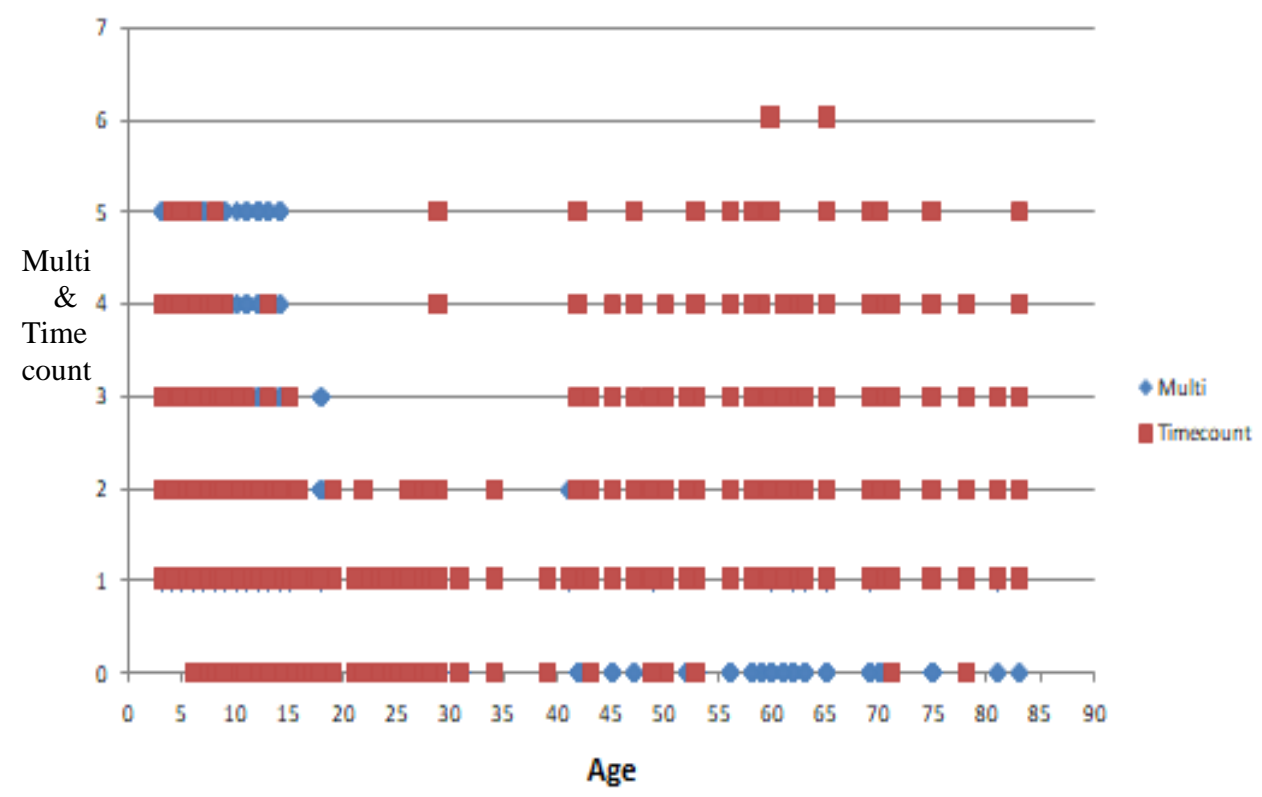

Fig. 6 Represents the multi is observed between 15 years and above 50 years

Here, in the Figure 6.1 we have represented in the graphical way as we have taken the field like age, multi touch and time count from the whole set of dataset. This takes the input of 10000 dataset and we can see that red square represents the time count of the individual age group persons and blue diamond represents the multi touch in the individual age group persons.

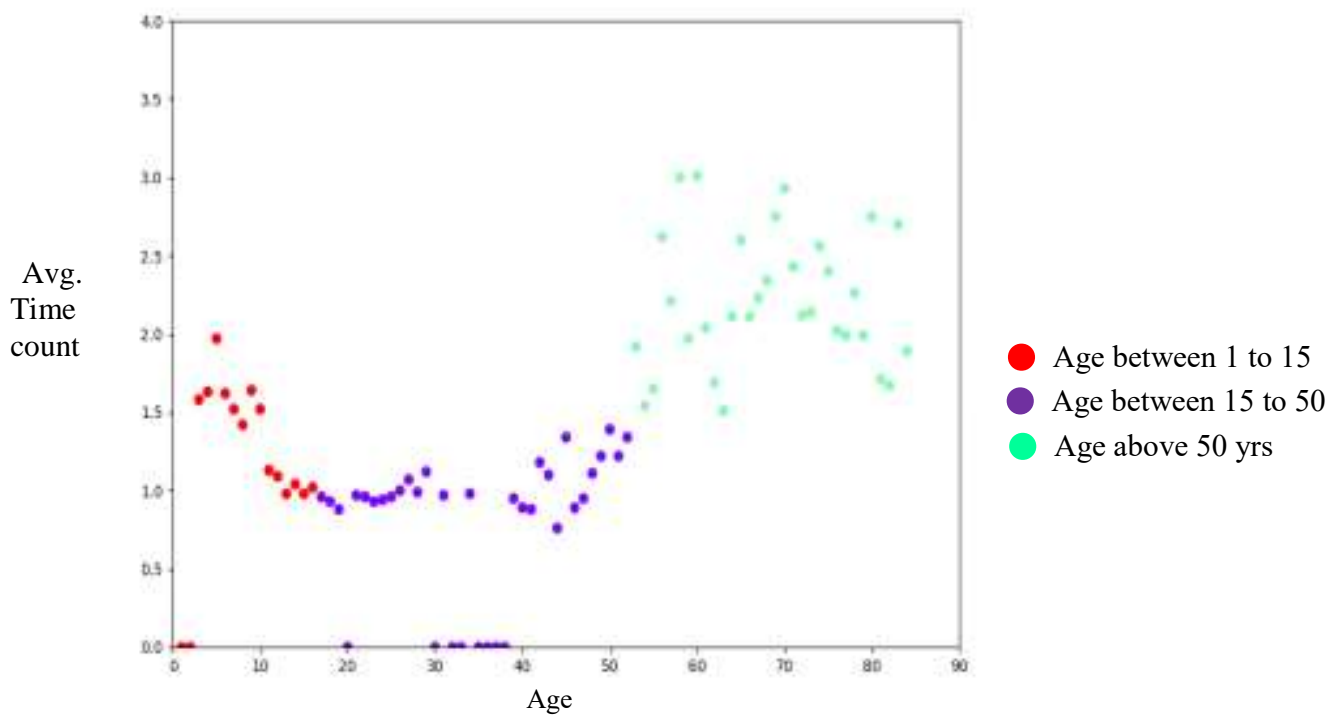

Fig. 7 Represents the average time count is observed above 50 years

From the Figure 5, we have applied K-means clustering algorithm to the dataset and obtained the clusters it is represented in Figure 6.2. In the Figure 6.2 red color dots represents the age group people between 1-15yrs, violet color dot represents the age 
group between the 15-50yrs and sea green color dot represents the age above 50yrs. As in the figure we can see that there is drastic increase in the time count from age above 50yrs. So, by this we can say that time count is occurred more in the age group above 50yrs.

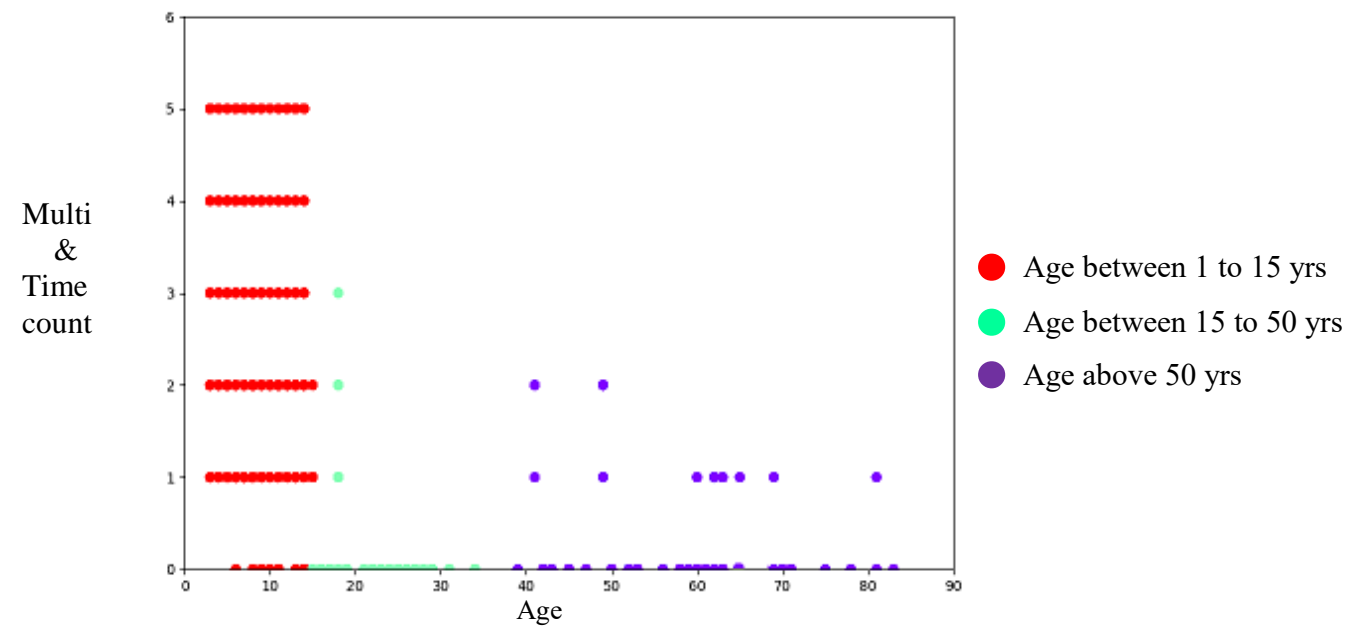

Fig. 8 Represents the multi and time count clustering

From the Figure 4 of the dataset we have taken the required fields as multi, time count and age. The required fields in the dataset are given to the K-Means Clustering algorithm from that we have obtained three clusters. Red color dots represent the age group people between 1-15yrs, sea green color dot represents the age group between the 15-50yrs and violet color dot represents the age above 50yrs. We can see from the figure that red color dots are constants this means that multi touch is occurred in the age group 1-15yrs.

\section{PREDICTION OF AGES}

From the above Clusters, we have generated some rules to determine the ages after creating clusters in which we have separated the age groups depending upon their touches and time elapsed between every single touch. By using this we can predict the specific age group of the person.

\begin{tabular}{|c|c|c|c|c|c|}
\hline Single & Multi & Xposition & Yposition & TimeCount & $\begin{array}{c}\text { Prediction } \\
\text { of Age }\end{array}$ \\
\hline 2 & 0 & 297.6799 & 70.46167 & 1 & $\mathbf{1 5 - 5 0}$ \\
\hline 3 & 1 & 384.5995 & 127.432 & 1 & $\mathbf{1 - 1 5}$ \\
\hline 4 & 2 & 233.7391 & 68.5752 & 1 & $\mathbf{1 - 1 5}$ \\
\hline 5 & 0 & 233.7391 & 68.5752 & 1 & $\mathbf{1 5 - 5 0}$ \\
\hline 6 & 0 & 185.7835 & 20.60022 & 2 & $>\mathbf{5 0}$ \\
\hline 7 & 0 & 854.209 & 1174.388 & 1 & $\mathbf{1 5 - 5 0}$ \\
\hline 8 & 0 & 295.6818 & 69.46228 & 3 & $>\mathbf{5 0}$ \\
\hline 9 & 3 & 881.1397 & 100.446 & 1 & $\mathbf{1 - 1 5}$ \\
\hline 10 & 0 & 904.1184 & 56.46899 & 1 & $\mathbf{1 5 - 5 0}$ \\
\hline
\end{tabular}

Fig. 8 Dataset for Predicting the Age

From this figure we have again developed the application without having the age field in it and we have extracted the data from the application. By this data considering our specification we can predict the age of the person. Like if multi touch is observed more than their age group is between 1-15yrs, if the time count is occurred more than their age group is above 50yrs. If multi touch and time count is not present than their age is between 15-50yrs. 
We have developed an application i.e., Android application in the application we touch on the screen at the front end, we get all the data like single touch, multi touch, Xposition, Y-position, age and time count. Here, we take only the field likes multi touch, age and time count. This data is obtained through XAMPP server. From the XAMPP server we retrieve the data. All this data is given to the K-Means Clustering Algorithm in order to obtain the clusters. From the Figure 6.1 we have represented in the graphical way as we have taken the field like age, multi touch and time count from the whole set of dataset. This takes the input of 10000 dataset and we can see that red square represents the time count of the individual age group persons and blue diamond represents the multi touch in the individual age group persons. By applying K-Means Algorithm we got the clusters as show in Figure 6.3.

Figure 5 represents the average time count of overall dataset according to their age groups this done by implementing using python code to it. By taking whole dataset and applying the python programming to the dataset we have chosen the field likes age and time count. To this dataset we have again applied K-Means Clustering Algorithm and obtained the three clusters and can see that sea green dots are observed and determine that there is drastic increase in time count for the age above 50yrs. According, to these three considerations we have again developed an application without the age field in it and we have extracted the data from the application. By this data considering our specifications we can predict the age of the person. Like if multi touch is observed more than their age group is between 1-15yrs, if the time count is occurred more than their age group is above $50 \mathrm{yrs}$. If multi touch and time count is not present than their age is between 15-50yrs.

\section{CONCLUSION}

We have built up a versatile application that takes the contribution from our fingers at the front end as a touch position and spares the server information. This data is given to the Machine Learning in order to train the data by using K- Means Clustering Algorithm, we then perform clustering by taking the average time count of the overall data and as well as, we have obtained clusters for the whole dataset. In average time count, we can observe there is a drastic increase in the time count value from the age above 50 years. Similarly in the whole dataset of age, multi-touch and time count, we can see that multitouch is more below 15 years age and sometimes it is observed that multi-touch appears above 50years. For the middle aged people multi touch and time count is not observed i.e., age between $15 \mathrm{yrs}$ to 50yrs. By using this data we can predict the person's age with the above considerations.

\section{REFERENCES}

[1] Zhaojian M., “Android-based Mobile Interlligent Application Development - The Development and Implementation of the Game Lianliankan[D]", Beijing University of Posts and Telecommunications, 2010.

[2] Developers A. What is Android[J]. 2011. https://developer.android.com/reference.

[3] XAMPP server installation https://pureinfotech.com/install-xampp-windows-10/.

[4] Server connection with the application "https://www.coderefer.com/android-localhost-connection/"

[5] Angra S and Ahuja S., "Machine Learning and its Applications: A Review", 2017 International Conference on the Big Data Analytics and Computational Intelligence (ICBDAC).

[6] Guo Jingfeng, Zhao Yuyan, Bian Weifeng, Li Jing, "A Hierarchical Clustering Algorithm Based on Cluster Cohesion and Separation", Journal of Computer Research and Development, (2009), pp. 202206.

[7] Xiong Wen, Jin Yaohong, "Hybrid Clustering Using ACO and AHC", Journal of Beijing University of Posts and Telecommunications, vol. 36.2013, pp. 60-63.

[8] Xu R, Wunsch D II, "Survey of clustering algorithms", IEEE Transaction on Neural Networks, Vol. 16, Issue 3, May 2005, pp. 645-678.

[9] Timothy J Ross, Fuzzy Logic with Engineering Applications, Mcgraw Hill, New York.

[10] Luai A. Shalabi, Zyad Shaaban and Basel Kassabeh, "Data Mining A Preprocessing Engine", Journal of Computer Science, Vol. 2, No. 9, 2006, pp. 735-739. 
[11] Visalakshi, N. K., \& Suguna, J. (2009). K-means clustering using Max-min distance measure. NAFIPS 2009 - 2009 Annual Meeting of the North American Fuzzy Information Processing Society.

[12] Ibrahim Mousa Al-Zuabi, Assef Jafar \& Kadan Aljoumaa, "Predicting customer's gender and age depending on mobile phone data", Journal of BigData, Vol.6, No.18, 2019, pp. 1-10.

[13] Nisreen Ameen and Robert Willis, "An analysis of the moderating effect of age on smartphone adoption and use in the United Arab Emirates", Proceedings for the UK Academy of Information Systems (UKAIS) conference 2018, At Oxford University, April, 2018, pp. 1-10.

[14] Leah Findlater, Jon E. Froehlich, Kays Fattal, Jacob O. Wobbrock, Tanya Dastyar, "Age-Related Differences in Performance with Touchscreens Compared to Traditional Mouse Input", ACM International Conference on Changing Perspectives, Paris, France, (2013), pp. 343-346.

[15] Asim Sinan Yuksel, Fatih Ahmet Senel, Ibrahim Arda Cankaya, "Classification of Soft Keyboard Typing Behaviors Using Mobile Device Sensors with Machine Learning”, April, 2019, vol. 44, no. 4, pp. 3929-3942.

[16] Nicholas D. Lane, Emiliano Miluzzo, Hong Lu, Daniel Peebles, Tanzeem Choudhury, and Andrew T. Campbell, Dartmouth College, "A Survey of Mobile Phone Sensing", IEEE Communication Magazines, 2010. 
International Journal of Control and Automation Vol. 12, No. 9 (2019) 